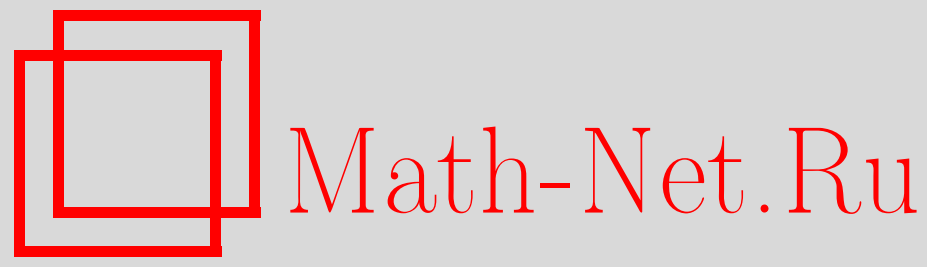

Л. Е. Посицельский, Алгебра замкнутых форм на диске кошулева, Функи. анализ и его прил., 2012, том 46, выпуск 3, 71-80

DOI: https://doi.org/10.4213/faa3078

Использование Общероссийского математического портала MathNet.Ru подразумевает, что вы прочитали и согласны с пользовательским соглашением

http://www . mathnet.ru/rus/agreement

Параметры загрузки:

IP : 54.197 .130 .99

26 апреля 2023 г., 02:46:15

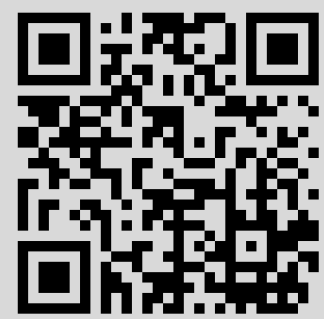


Функционалъный анализ и его приложения

2012, т. 46, вып. 3, с. 71-80

УДК $514.7+512.66$

\title{
Алгебра замкнутых форм на диске кошулева*
}

\author{
(c) 2012. Л. Е. ПосицЕЛЬСКий
}

В работе показано, что алгебра замкнутых дифференциальных форм на (алгебраическом, формальном или аналитическом) диске с логарифмическими особенностями вдоль нескольких координатных гиперплоскостей является (как нетопологически, так и топологически) кошулевой. Связь с вариациями смешанных структур Ходжа-Тейта обсуждается во введении.

\section{Введение}

В настоящей работе рассматриваются алгебры замкнутых дифференциальных форм на диске, регулярных вне нескольких выбранных координатных гиперплоскостей и имеющих, самое большее, логарифмические особенности вдоль этих гиперплоскостей, по отношению к операции умножения дифференциальных форм. Такие алгебры возникают при изучении смешанных пучков ХоджаТейта на гладких алгебраических многообразиях [2]. Точнее говоря, указанные алгебры замкнутых форм на диске играют роль в локальном описании таких пучков в окрестности точки, которая может принадлежать либо исходному многообразию, либо дивизору с нормальными пересечениями, образующему бесконечно удаленную часть его гладкой компактификации.

Пусть $D$ - комплексно-аналитический диск и $V$ - дополнение к нескольким координатным гиперплоскостям в $D$. Согласно результатам работы [2], вещественные смешанные пучки Ходжа-Тейта на $V$ с допустимыми особенностями в $D \backslash V$ описываются в терминах ассоциативной суперкоммутативной положительно внутренне градуированной DG-алгебры $\mathbb{R} \mathcal{H} \mathcal{T}_{(V, D)}$. Когомологии DG-алгебры $\mathbb{R} \mathcal{H} \mathcal{T}_{(V, D)}$ лежат на объединении двух полупрямых: диагонали, где внутренняя градуировка равна когомологической, и оси, на которой когомологическая градуировка равна единице. Диагональная часть когомологий изоморфна алгебре замкнутых форм на $V$ с логарифмическими особенностями вдоль $D \backslash V$, в то время как часть, лежащая на второй полупрямой (отвечающая за смешанные структуры Ходжа над точкой), имеет одномерную компоненту в каждой положительной внутренней градуировке.

Коммутативная алгебра Хопфа, описывающая категорию смешанных пучков Ходжа-Тейта на $(V, D)$ в рамках таннакиева формализма, есть алгебра нулевых когомологий приведенной бар-конструкции $\mathrm{DG}$-алгебры $\mathbb{R} \mathcal{H} \mathcal{T}_{(V, D)}$. Из кошулевости алгебры замкнутых форм, доказанной в настоящей работе, следует, что эта бар-конструкция не имеет когомологий в когомологических градуировках,

* Автор получал финансовую поддержку гранта из средств премии Бальзана, полученной П. Делинем в 2004 году, гранта фонда Саймонса и грантов РФФИ 10-01-93113-НЦНИЛ_а, 1101-00393-а, 11-01-12072-офи-м-2011 во время работы над окончательной версией аргументов и написания статьи. 
отличных от нуля. Чтобы убедиться в этом, достаточно рассмотреть спектральную последовательность, сходящуюся от когомологий бар-конструкции алгебры когомологий DG-алгебры $\mathbb{R} \mathcal{H} \mathcal{T}_{(V, D)}$ к когомологиям бар-конструкции самой $\mathrm{DG}$-алгебры $\mathbb{R} \mathcal{H} \mathcal{T}_{(V, D)}$, и воспользоваться известным описанием алгебры Ext для связной прямой суммы аугментированных алгебр [5, предложение 1.1 гл. 3]. Коммутативная алгебра Хопфа нулевых когомологий является косвободным произведением алгебры Хопфа, квадратично двойственной к алгебре замкнутых дифференциальных форм, и косвободной алгебры Хопфа с однородными кообразующими, занумерованными натуральными числами.

Различные свойства кошулевости алгебр мотивных когомологий (милноровской К-теории, когомологий Галуа и т.д.) играют важную роль в теории мотивов (см. [3], [4] и другие работы автора на данную тему; рассуждения, приведенные выше, являются новой иллюстрацией этого общего наблюдения). Однако в настоящее время наличие таких свойств является по большей части гипотезами, а не теоремами. Алгебры замкнутых дифференциальных форм на диске, рассматриваемые в настоящей работе, представляют собой интересное семейство алгебр, имеющих мотивное значение, кошулевость которых оказывается легко установить. Это и будет продемонстрировано ниже.

Автор благодарен А. М. Левину за постановку задачи. Эта работа была в основном выполена, когда А. М. Л. и я оба были приглашены в Математический институт Макса Планка в Бонне весной 2003 года, и я хотел бы поблагодарить институт за гостеприимство.

\section{§1. Модульная кошулевость}

Пусть $D$ - диск с координатами $z_{1}, \ldots, z_{u}$. За немногими исключениями для нас не будет иметь значения, какая конкретно геометрическая категория подразумевается. Так, $D$ может быть алгебраическим аффинным пространством над полем нулевой характеристики, формальным диском над таким полем, комплексно-аналитическим или вещественно-гладким диском. Можно также взять за $D$ спектр алгебры многочленов или формальных степенных рядов с разделенными степенями над полем простой характеристики.

Пусть $0 \leqslant v \leqslant u$. Для любого $1 \leqslant s \leqslant v$ пусть $L_{s}$ обозначает координатную гиперплоскость $\left\{z_{s}=0\right\} \subset D$. Обозначим через $\Omega$ DG-алгебру де Рама регулярных дифференциальных форм на $D \backslash \bigcup_{s=1}^{v} L_{s}$ с логарифмическими особенностями вдоль $L_{s}$. Пусть $Z \subset \Omega$ - подалгебра замкнутых форм, т. е. ядро дерамовского дифференциала $d: \Omega \rightarrow \Omega$. Пусть $H=H(\Omega, d)$ - алгебра когомологий DG-алгебры $\Omega$.

Обозначим через $A$ внешнюю алгебру, порожденную замкнутыми 1-формами $d z_{s} / z_{s}, s \leqslant v$, и $d z_{r}, r>v$. Для нас важно только контролировать гомологические свойства $A$-модулей $\Omega$ и $H$. В частности, можно заменить диск на любое пространство, этально отображающееся в диск и удовлетворяющее подходящей версии леммы Пуанкаре. В примерах выше $\Omega$ является свободным $A$-модулем, порожденным $\Omega^{0}$, и $H$ есть внешняя алгебра, порожденная формами $d z_{s} / z_{s}$, с очевидной структурой $A$-модуля, в которой $d z_{s} / z_{s} \in A$ действуют свободно и $d z_{r} \in A$ действуют тривиально на $H$.

Понятие кошулевой алгебры, введенное Придди [6] в контексте алгебр, локально конечномерных относительно дополнительной градуировки, и больше всего изучавшееся для алгебр с конечномерным пространством образующих [5], 
можно без труда обобщить на полностью бесконечномерный случай [3]. То же относится к понятию кошулева модуля [4], введенному Бейлинсоном, Гинзбургом и Зёргелем [1]. Основная разница с локально конечномерным случаем состоит в том, что в бесконечномерной ситуации квадратичная двойственность связывает алгебры и коалгебры, а также модули и комодули.

Напомним эти определения. Неотрицательно градуированная алгебра $A$ над полем $k$ называется кошулевой, если $A_{0}=k$ и $\operatorname{Tor}_{i j}^{A}(k, k)=0$ для $i \neq j$. Kогда алгебра $A$ кошулева, неотрицательно градуированный левый $A$-модуль $M$ называется кошулевым, если $\operatorname{Tor}_{i j}^{A}(k, M)=0$ для $i \neq j$. Здесь первый индекс $i$ обозначает гомологическую градуировку пространств Tor, а второй индекс $j$ - внутреннюю градуировку (см. работы, упоминавшиеся выше). Отметим, что для любых неотрицательно градуированных алгебры $A$ и модуля $M$ из условия $A_{0}=k$ следует, что $\operatorname{Tor}_{i j}^{A}(k, k)=0=\operatorname{Tor}_{i j}^{A}(k, M)$ для $i>j$. Мы будем использовать верхние и нижние индексы взаимозаменяемым образом для обозначения внутренних градуировок; никаких изменений знаков при переходе от верхних к нижним индексам и обратно не подразумевается.

Лемма. Пусть $M$ - неотрицательно градуированный левый модуль над кошулевой алгеброй $A$. Тогда градуированное векторное пространство $\operatorname{Tor}_{i}^{A}(k, M)$ сосредоточено в градуировках $i$ u $i+1$ для всех $i$, если и только если $M_{+}=$ $M_{1} \oplus M_{2} \oplus \cdots$ - кошулев левый $A$-модуль в градуировке, сдвинутой на 1 ( так, чтобы компонента $M_{1}$ попала в градуировку 0$)$.

Доказательство. Утверждение следует из длинной точной последовательности $\cdots \rightarrow \operatorname{Tor}_{i+1}^{A}\left(k, M_{0}\right) \rightarrow \operatorname{Tor}_{i}^{A}\left(k, M_{+}\right) \rightarrow \operatorname{Tor}_{i}^{A}(k, M) \rightarrow \operatorname{Tor}_{i}^{A}\left(k, M_{0}\right) \rightarrow \cdots$, поскольку градуированное векторное пространство $\operatorname{Tor}_{i}^{A}\left(k, M_{0}\right)$ сосредоточено в градуировке $i$ (в силу кошулевости алгебры $A$ ).

Теорема 1. Пусть $(\Omega, d)$ - неотрицательно градуированная DG-алгебра над полем $k$ с дифберенииалом степени 1 ; положим $Z=\operatorname{ker} d u H=H(\Omega, d)$. Пусть $A$ - кошулева алгебра и $f: A \rightarrow Z$ - морбизм градуированных алгебр. Предположим, что $\Omega$ и $H$ - кошулевы левые $A$-модули по отношению $к$ модульным структурам, индуиированным морфизмом $f$. Тогда $Z^{+}=Z^{1} \oplus Z^{2} \oplus \cdots$ - кошулев левый $A$-модуль в градуировке, сдвинутой на 1.

Доказательство. Для любого градуированного $A$-модуля $M$ и $j \in \mathbb{Z}$ обозначим через $M(j)$ градуированный $A$-модуль с компонентами $M(j)^{m}=M^{m-j}$ и действием алгебры $A$, определенным по правилу $a \cdot x(j)=(-1)^{j n}(a \cdot x)(j)$ для $x \in M$ и $a \in A^{n}$. Рассмотрим комплекс градуированных $A$-модулей

$$
C: \cdots \longrightarrow \Omega(3) \longrightarrow \Omega(2) \longrightarrow \Omega(1) \longrightarrow Z .
$$

Нас интересуют две спектральные последовательности гипергомологий с одним и тем же пределом, получающиеся при применении производного функтора $\operatorname{Tor}^{A}(k,-)$ к этому комплексу $C$. Конкретно, мы имеем ' $E_{p q}^{2},{ }^{\prime \prime} E_{p q}^{1} \Longrightarrow$ $\operatorname{Tor}_{p+q}^{A}(k, C)$, где ' $E_{p q}^{2}=\operatorname{Tor}_{p}^{A}(k, H(q))$ и ${ }^{\prime \prime} E_{p q}^{1}=\operatorname{Tor}_{q}^{A}(k, \Omega(p))$ для $p>0$, в то время как ${ }^{\prime \prime} E_{0, q}^{1}=\operatorname{Tor}_{q}^{A}(k, Z)$.

По предположению член ' $E_{p q}^{2}$ сосредоточен во внутренней градуировке $p+q$, так что предельный член $\operatorname{Tor}_{i}^{A}(k, C)$ сосредоточен во внутренней градуировке $i$. Далее, член ${ }^{\prime \prime} E_{p q}^{1}$ сосредоточен во внутренней градуировке $p+q$ для всех $p>0$. 
Теперь все компоненты члена " $E_{0, i}^{1}$ внутренней градуировки, отличной от $i$, должны убиваться дифференциалами ${ }^{\prime \prime} d^{r}:{ }^{\prime \prime} E_{r, i-r+1}^{r} \rightarrow{ }^{\prime \prime} E_{0, i}^{r}$, так что член ${ }^{\prime \prime} E_{0, i}^{1}$ сосредоточен во внутренних градуировках $i$ и $i+1$. Остается воспользоваться леммой.

Для неотрицательно градуированной $k$-алгебры $B$ положим $B^{\prime}=k \oplus B_{1} \oplus$ $B_{2} \oplus \cdots$. Согласно предыдущей лемме и [4, теорема 6.1], если $A \rightarrow B^{\prime}$ - гомоморфизм градуированных алгебр, алгебра $A$ кошулева и $B_{+}-$кошулев левый $A$-модуль в градуировке, сдвинутой на 1 , то алгебра $B^{\prime}$ кошулева. (Теорема 2 из $\S 3$ доставляет другое доказательство того же результата.) Поэтому в предположениях теоремы все три градуированные алгебры $\Omega^{\prime}, H^{\prime}$ и $Z^{\prime}$ кошулевы.

Следствие. В любой из перечисленных выше геометрических категорий алгебра $Z$ замкнутых дифференииальных форм на диске $D$ с логарифмическими особенностями вдоль нескольких координатных гиперплоскостей $L_{s}$ является кошулевой алгеброй.

Доказательство. Как видно из обсуждения в начале параграфа, алгебра $\Omega$ логарифмических дифференциальных форм на $D$ относительно $\left\{L_{s}\right\}$ и ее алгебра дерамовских когомологий $H$ являются кошулевыми модулями над внешней алгеброй $A$, порожденной $d z_{s} / z_{s}$ и $d z_{r}$. Кроме того, $Z^{0}=k$. Поэтому утверждение следствия вытекает из доказанной выше теоремы 1 и теоремы 6.1 из работы [4].

\section{§2. Замечания о топологической кошулевости}

Кошулевость градуированной алгебры $Z$ - это условие точности бар-комплекса этой алгебры, компоненты которого суть прямые суммы тензорных произведений градуировочных компонент алгебры $Z$. Однако тензорные произведения градуировочных компонент алгебры $Z$ не всегда являются естественным объектом для рассмотрения. Совершенно естественно рассматривать такие тензорные произведения, когда $Z$ есть алгебра функций или форм на алгебраическом многообразии, но, возможно, не в том случае, когда $Z$ есть алгебра форм на формальном, комплексно-аналитическом или вещественно-гладком диске. В последних случаях, может быть, лучше рассматривать пополненные тензорные произведения. Отметим, что, в частности, в задаче о локальном описании смешанных пучков Ходжа-Тейта возникает алгебра форм на комплексноаналитическом диске (см. введение к настоящей работе и препринт [2]).

Конкретно, может быть желательным определить пополненное тензорное произведение $Z_{n_{1}} \widehat{\otimes} \cdots \widehat{\otimes} Z_{n_{m}}$ как пространство замкнутых форм на $D^{m}$ степени $n_{t}$ по отношению к $t$-й группе переменных с логарифмическими особенностями вдоль гиперплоскостей $D^{t} \times L_{s} \times D^{m-t-1}$. После этого можно построить пополненный бар-комплекс из таких пополненных тензорных произведений и задаться вопросом о его точности вне диагонали. Более того, можно рассматривать диагональные гомологии этого комплекса как пополненную версию коалгебры, кошулево двойственной к $Z$. Компонента степени $n$ этой пополненной коалгебры есть пространство всех замкнутых форм на $D^{n}$ степени 1 по отношению к каждой группе переменных с зануляющимися обратными образами относительно отображений $\operatorname{Id}_{D}^{t-1} \times \Delta_{D} \times \operatorname{Id}_{D}^{n-t-1}: D^{n-1} \rightarrow D^{n}$, где $\Delta_{D}: D \rightarrow D^{2}$ обозначает диагональное отображение. 
С точки зрения специалиста по гомологической алгебре топологическая алгебра как таковая - это зыбкая почва, которой лучше по возможности избегать. Поэтому мы предлагаем простой линейно-алгебраический формализм для описания топологической кошулевости в указанном выше смысле, не зависящий ни от какого понятия топологического тензорного произведения.

С любой ассоциативной алгеброй $Z$ с единицей над полем $k$ можно связать семейство векторных пространств $Z_{n}=Z^{\otimes n}, n \geqslant 0$, снабженных линейными отображениями, индуцированными умножением и единицей в $Z$. Структура, которая таким образом получается, - это симплициальное $k$-векторное пространство $Z_{\bullet}$, снабженное отображением $k \rightarrow Z \bullet$ в него из постоянного симплициального векторного пространства $k$.

Когда $Z$ - градуированная алгебра, получается гораздо более богатая структура. (Квазиассоциативная градуированная) квазиалгебра $\mathcal{Z}$ над полем $k$ - это семейство векторных пространств $\mathcal{Z}_{n_{1}, \ldots, n_{m}}$, где $m \geqslant 0$ и $n_{t} \in \mathbb{Z}$, снабженных отображениями квазиумножения

$$
\mathcal{Z}_{n_{1}, \ldots, n_{m}} \longrightarrow \mathcal{Z}_{n_{1}, \ldots, n_{t-1}, n_{t}+n_{t+1}, n_{t+2}, \ldots, n_{m}}
$$

и отображениями квазиединищъ

$$
\mathcal{Z}_{n_{1}, \ldots, n_{m}} \longrightarrow \mathcal{Z}_{n_{1}, \ldots, n_{t}, 0, n_{t+1}, \ldots, n_{m}}
$$

обладающими обычным свойствам отображений умножения и единицы между тензорными произведениями $\mathcal{Z}_{n_{1}, \ldots, n_{m}}=Z_{n_{1}} \otimes_{k} \cdots \otimes_{k} Z_{n_{m}}$ градуировочных компонент ассоциативной алгебры с единицей. Конкретнее, композиции отображений

$$
\mathcal{Z}_{n_{1}, \ldots, n_{m}} \longrightarrow \mathcal{Z}_{n_{1}, \ldots, n_{s}+n_{s+1}, \ldots, n_{t}, \ldots, n_{m}} \longrightarrow \mathcal{Z}_{n_{1}, \ldots, n_{s}+n_{s+1}, \ldots, n_{t}+n_{t+1}, \ldots, n_{m}}
$$

и

$$
\mathcal{Z}_{n_{1}, \ldots, n_{m}} \longrightarrow \mathcal{Z}_{n_{1}, \ldots, n_{s}, \ldots, n_{t}+n_{t+1}, \ldots, n_{m}} \longrightarrow \mathcal{Z}_{n_{1}, \ldots, n_{s}+n_{s+1}, \ldots, n_{t}+n_{t+1}, \ldots, n_{m}}
$$

должны совпадать; композиции отображений

$$
\mathcal{Z}_{n_{1}, \ldots, n_{m}} \longrightarrow \mathcal{Z}_{n_{1}, \ldots, n_{t-1}+n_{t}, \ldots, n_{m}} \longrightarrow \mathcal{Z}_{n_{1}, \ldots, n_{t-1}+n_{t}+n_{t+1}, \ldots, n_{m}}
$$

и

$$
\mathcal{Z}_{n_{1}, \ldots, n_{m}} \longrightarrow \mathcal{Z}_{n_{1}, \ldots, n_{t}+n_{t+1}, \ldots, n_{m}} \longrightarrow \mathcal{Z}_{n_{1}, \ldots, n_{t-1}+n_{t}+n_{t+1}, \ldots, n_{m}}
$$

должны совпадать. Отображения квазиединицы должны коммутировать с отображениями квазиумножения; композиции

$$
\mathcal{Z}_{n_{1}, \ldots, n_{m}} \longrightarrow \mathcal{Z}_{n_{1}, \ldots, n_{t}, 0, n_{t+1}, \ldots, n_{m}} \longrightarrow \mathcal{Z}_{n_{1}, \ldots, n_{t}+0, n_{t+1}, \ldots, n_{m}}=\mathcal{Z}_{n_{1}, \ldots, n_{m}}
$$

и

$$
\mathcal{Z}_{n_{1}, \ldots, n_{m}} \longrightarrow \mathcal{Z}_{n_{1}, \ldots, n_{t}, 0, n_{t+1}, \ldots, n_{m}} \longrightarrow \mathcal{Z}_{n_{1}, \ldots, n_{t}, 0+n_{t+1}, \ldots, n_{m}}=\mathcal{Z}_{n_{1}, \ldots, n_{m}}
$$

должны быть тождественными отображениями. Компонента $\mathcal{Z}_{\varnothing}$ с $m=0$ индексами должна быть отождествлена с $k$ (ср. [5, §4 гл. 3]).

Квазиалгебра называется неотрицательной, если $\mathcal{Z}_{n_{1}, \ldots, n_{m}}=0$, как только $n_{t}<0$ для некоторого $1 \leqslant t \leqslant m$. Неотрицательная квазиалгебра называется положительной, если все ее отображения квазиединицы являются изоморфизмами. Положительная квазиалгебра определяется своими компонентами $\mathcal{Z}_{n_{1}, \ldots, n_{m}}$ с положительными индексами $n_{m}>0$ и отображениями квазиумножения между ними, удовлетворяющими уравнениям квазиассоциативности 
(т. е. тем из сформулированных выше уравнений, которые зависят только от отображений квазиумножения).

Пусть $Z$ - положительно градуированная ассоциативная алгебра над $k$, т. е. $Z$ неотрицательно градуирована в очевидном смысле и $Z_{0}=k$. Положим $Z_{+}=$ $Z / k$. Тогда можно ассоциировать с $Z$ ее приведенный бар-комплекс $B$ вида

$$
k \longleftarrow Z_{+} \longleftarrow Z_{+} \otimes_{k} Z_{+} \longleftarrow Z_{+} \otimes_{k} Z_{+} \otimes_{k} Z_{+} \longleftarrow \cdots,
$$

рассмотреть его градуировочную компоненту $B_{n}$ внутренней градуировки $n$ и взять тензорное произведение $\mathcal{B}_{n_{1}, \ldots, n_{m}}=B_{n_{1}} \otimes_{k} \cdots \otimes_{k} B_{n_{m}}$ нескольких таких комплексов. Компоненты комплекса $\mathcal{B}_{n_{1}, \ldots, n_{m}}$ суть прямые суммы тензорных произведений градуировочных компонент алгебры $Z$. Заменив все такие тензорные произведения компонентами $\mathcal{Z}_{n_{1}^{\prime}, \ldots, n_{m^{\prime}}^{\prime}}$ произвольной положительной квазиалгебры $\mathcal{Z}$, можно определить комплекс $\mathcal{B}_{n_{1}, \ldots, n_{m}}$ как аддитивный функтор на абелевой категории положительных квазиалгебр. Положительная квазиалгебра $\mathcal{Z}$ называется кошулевой, если все комплексы $\mathcal{B}_{n_{1}, \ldots, n_{m}}$ имеют гомологии только в гомологической степени $n_{1}+\cdots+n_{m}$.

Обращая все стрелки в приведенных выше определениях, получаем определения (квазикоассоциативных градуированных) квазикоалгебр и, в частности, положительных квазикоалгебр и кошулевых квазикоалгебр. В частности, квазикоумножения суть отображения $\mathcal{C}_{n_{1}, \ldots, n_{t-1}, n_{t}+n_{t+1}, n_{t+2}, \ldots, n_{m}} \longrightarrow \mathcal{C}_{n_{1}, \ldots, n_{m}}$, квазикоединицы суть отображения $\mathcal{C}_{n_{1}, \ldots, n_{t}, 0, n_{t+1}, \ldots, n_{m}} \longrightarrow \mathcal{C}_{n_{1}, \ldots, n_{m}}$, и кошулевость положительных квазикоалгебр определяется в терминах комплексов, имитирующих тензорные произведения градуировочных компонент приведенных кобаркомплексов положительно градуированных коалгебр.

Аддитивные категории кошулевых квазиалгебр и кошулевых квазикоалгебр эквивалентны. Функтор эквивалентности сопоставляет кошулевой квазиалгебре $\mathcal{Z}$ кошулеву квазикоалгебру $\mathcal{C}$ с компонентами $\mathcal{C}_{n_{1}, \ldots, n_{m}}=H_{n_{1}+\cdots+n_{m}}\left(\mathcal{B}_{n_{1}, \ldots, n_{m}}\right)$. Естественные сюръективные отображения комплексов

$$
\mathcal{B}_{n_{1}, \ldots, n_{t-1}, n_{t}+n_{t+1}, n_{t+2}, \ldots, n_{m}} \longrightarrow \mathcal{B}_{n_{1}, \ldots, n_{m}}
$$

индуцируют отображения квазикоумножения на $\mathcal{C}$. Обратный функтор определяется аналогичным образом в терминах кобар-комплексов квазикоалгебр.

В частности, все отображения квазиумножения между компонентами с неотрицательными индексами у кошулевой квазиалгебры сюръективны. Это аналог условия порожденности градуированной алгебры $Z$ компонентой $Z_{1}$ для случая квазиалгебр. Есть также аналог условия квадратичности и двойственные условия для кошулевых квазикоалгебр. В силу этих условий двойственные кошулевы квазиалгебра $\mathcal{Z}$ и квазикоалгебра $\mathcal{C}$ однозначно определяются векторными пространствами $\mathcal{Z}_{1, \ldots, 1} \simeq \mathcal{C}_{1, \ldots, 1}$ и точными последовательностями

$$
0 \longrightarrow \mathcal{C}_{1, \ldots, 1,2,1, \ldots, 1} \longrightarrow \mathcal{C}_{1, \ldots, 1} \simeq \mathcal{Z}_{1, \ldots, 1} \longrightarrow \mathcal{Z}_{1, \ldots, 1,2,1, \ldots, 1} \longrightarrow 0 .
$$

Чтобы соответствующие квазикоалгебра $\mathcal{C}$ и квазиалгебра $\mathcal{Z}$ были кошулевыми, набор из $n-1$ подпространств $\mathcal{C}_{1, \ldots, 1,2,1, \ldots, 1}$ в векторном пространстве $\mathcal{C}_{1, \ldots, 1}$ ( $n$ единиц) должен быть дистрибутивным для всех $n$ (ср. $[3, \S 2.2])$.

Квазиалгебра с внешними умножениями - это квазиалгебра $\mathcal{Z}$, снабженная линейными отображениями

$$
\mathcal{Z}_{n_{1}, \ldots, n_{t}} \otimes_{k} \mathcal{Z}_{n_{t+1}, \ldots, n_{m}} \longrightarrow \mathcal{Z}_{n_{1}, \ldots, n_{m}}
$$


совместимыми с отождествлением $\mathcal{Z}_{\varnothing}=k$ и коммутирующими с отображениями квазиумножения и квазиединицы. Квазикоалгебры с внешними умножениями определяются аналогично. На этот раз стрелки не обращаются, т. е. внешние умножения в квазикоалгебре имеют вид

$$
\mathcal{C}_{n_{1}, \ldots, n_{t}} \otimes_{k} \mathcal{C}_{n_{t+1}, \ldots, n_{m}} \longrightarrow \mathcal{C}_{n_{1}, \ldots, n_{m}}
$$

Квазиалгебра или квазикоалгебра, соответствующая градуированной алгебре или коалгебре, естественным образом снабжена внешними умножениями, и квази(ко)алгебра с внешними умножениями происходит из (однозначно определенной) градуированной (ко)алгебры тогда и только тогда, когда все ее отображения внешнего умножения являются изоморфизмами.

Эквивалентность между категориями кошулевых квазиалгебр и кошулевых квазикоалгебр преобразует квазиалгебры с внешними умножениями в квазикоалгебры с внешними умножениями и обратно. Чтобы убедиться в этом, достаточно построить естественные внешние умножения

$$
\mathcal{B}_{n_{1}, \ldots, n_{t}} \otimes_{k} \mathcal{B}_{n_{t+1}, \ldots, n_{m}} \longrightarrow \mathcal{B}_{n_{1}, \ldots, n_{m}}
$$

на бар-комплексах квазиалгебры с внешними умножениями и аналогичные внешние умножения на кобар-комплексах квазикоалгебры с внешними умножениями.

\section{§3. Модульная кошулевость для квазиалгебр}

Пусть $A$ - кошулева алгебра и $\mathcal{Z}$ - положительная квазиалгебра над полем $k$. Предположим, что $A$ действует слева на $\mathcal{Z}$ в следующем смысле: для любых $l_{p}, \ldots, l_{1}, n_{0}, \ldots, n_{q} \geqslant 0, p, q \geqslant 0$, имеются линейные отображения

$$
A_{n} \otimes_{k} \mathcal{Z}_{l_{p}, \ldots, l_{1}, n_{0}, \ldots, n_{q}} \longrightarrow \mathcal{Z}_{l_{p}, \ldots, l_{1}, n+n_{0}, n_{1}, \ldots, n_{q}},
$$

превращающие $\bigoplus_{n_{0}=0}^{\infty} \mathcal{Z}_{l_{p}, \ldots, l_{1}, n_{0}, \ldots, n_{q}}$ в градуированный левый $A$-модуль для любых фиксированных $l_{p}, \ldots, l_{1}, n_{1}, \ldots, n_{q}$ и коммутирующие с отображениями квазиумножения

$$
\mathcal{Z}_{l_{p}, \ldots, l_{1}, n_{0}, \ldots, n_{q}} \longrightarrow \mathcal{Z}_{l_{p}, \ldots, l_{1}, n_{0}, \ldots, n_{t}+n_{t+1}, \ldots, n_{q}}
$$

для всех $0 \leqslant t<q$.

Для любой положительно градуированной ассоциативной алгебры $Z$ над $k$ рассмотрим тензорное произведение $E_{l_{p}, \ldots, l_{0} ; n_{1}, \ldots, n_{q}}=Z_{l_{p}} \otimes_{k} \cdots \otimes_{k} Z_{l_{0}} \otimes_{k} B_{n_{1}} \otimes_{k}$ $\cdots \otimes_{k} B_{n_{q}}$ градуировочных компонент алгебры $Z$ и ее приведенного бар-комплекса $B$ (с тривиальными коэффициентами). Члены комплекса $E_{l_{p}, \ldots, l_{0} ; n_{1}, \ldots, n_{q}}$ суть прямые суммы тензорных произведений градуировочных компонент алгебры $Z$; заменив все такие тензорные произведения компонентами $\mathcal{Z}_{n_{1}^{\prime}, \ldots, n_{m^{\prime}}^{\prime}}$

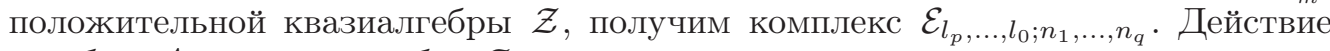
алгебры $A$ на квазиалгебре $\mathcal{Z}$ индуцирует ее действие

$$
A_{n} \otimes_{k} \mathcal{E}_{l_{p}, \ldots, l_{0} ; n_{1}, \ldots, n_{q}} \longrightarrow \mathcal{E}_{l_{p}, \ldots, l_{1}, n+l_{0} ; n_{1}, \ldots, n_{q}}
$$

на комплексах $\mathcal{E}_{l_{p}, \ldots, l_{0} ; n_{1}, \ldots, n_{q}}$, а следовательно, и на их когомологиях.

Теорема 2. Предположим, что градуированный $A$-модуль

$$
\bigoplus_{l_{0}=1}^{\infty} H_{n_{1}+\cdots+n_{q}}\left(\mathcal{E}_{l_{p}, \ldots, l_{0} ; n_{1}, \ldots, n_{q}}\right)
$$


кошулев в градуировке, сдвинутой на 1 , для любых фиксированных $l_{p}, \ldots, l_{1}, n_{1}$, $\ldots, n_{q} \geqslant 1, p, q \geqslant 0$. Тогда квазиалгебра $\mathcal{Z}$ кошулева.

Доказательство. Покажем, что гомологии комплекса $\mathcal{E}_{l_{p}, \ldots, l_{1} ; n_{0}, \ldots, n_{q}}$ сосредоточены в градуировке $n_{0}+\cdots+n_{q}$ для всех $l_{p}, \ldots, l_{1}, n_{0}, \ldots, n_{q} \geqslant 1, p, q \geqslant 0$. Воспользуемся индукцией по $n_{0}+\cdots+n_{q}$.

Представим себе, что имеется морфизм положительно градуированных алгебр $A \rightarrow Z$, и рассмотрим бикомплекс $F=\bigoplus_{t^{\prime}, t^{\prime \prime}=0}^{\infty} A_{+}^{\otimes t^{\prime}} \otimes_{k} Z \otimes_{k} Z_{+}^{\otimes t^{\prime \prime}}$ с одним дифференциалом, индуцированным дифференциалом приведенного баркомплекса алгебры $A$ с коэффициентами в левом $A$-модуле $Z$, и другим, индуцированным дифференциалом приведенного бар-комплекса алгебры $Z$ с коэффициентами в правом $Z$-модуле $Z$. Рассмотрим компоненту $F_{n_{0}}$ комплекса $F$ (полной) внутренней градуировки $n_{0}$ и возьмем ее тензорное произведение $Z_{l_{p}} \otimes_{k} \cdots \otimes_{k} Z_{l_{1}} \otimes_{k} F_{n_{0}} \otimes_{k} B_{n_{1}} \otimes_{k} \cdots \otimes_{k} B_{n_{q}}$ с компонентами алгебры $Z$ и ее приведенного бар-комплекса $B$ (с тривиальными коэффициентами). Обозначим

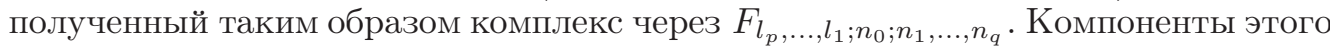
комплекса суть прямые суммы тензорных произведений градуировочных компонент алгебр $A$ и $Z$. Перенося тензорные произведения компонент алгебры $A$ налево в этих тензорных произведениях и заменяя все тензорные произведения компонент алгебры $Z$ компонентами $\mathcal{Z}_{n_{1}^{\prime}, \ldots, n_{m^{\prime}}^{\prime}}$ положительной квазиалгебры $\mathcal{Z}$, получаем комплекс $\mathcal{F}_{l_{p}, \ldots, l_{1} ; n_{0} ; n_{1}, \ldots, n_{q}}$, зависящий как аддитивный функтор от квазиалгебры $\mathcal{Z}$ с левым действием $A$.

Используя версию стягивающей гомотопии бар-комплекса алгебры $Z$ с коэффициентами в $Z$ для случая квазиалгебр, индуцированную изоморфизмами $\mathcal{Z}_{l_{p}, \ldots, l_{1}, n_{0}^{\prime}, \ldots, n_{q^{\prime}}^{\prime}} \rightarrow \mathcal{Z}_{l_{p}, \ldots, l_{1}, 0, n_{0}^{\prime}, \ldots, n_{q^{\prime}}}$, можно видеть, что комплекс $\mathcal{F}_{l_{p}, \ldots, l_{1} ; n_{0} ; n_{1}, \ldots, n_{q}}$ квазиизоморфен тензорному произведению компоненты внутренней градуировки $n_{0}$ приведенного бар-комплекса алгебры $A$ (с тривиальными коэффициентами) и комплекса $\mathcal{E}_{l_{p}, \ldots, l_{1} ; n_{1}, \ldots, n_{q}}$. Поэтому из предположения индукции следует, что гомологии $\mathcal{F}_{l_{p}}, \ldots, l_{1} ; n_{0} ; n_{1}, \ldots, n_{q}$ сосредоточены в гомологической градуировке $n_{0}+\ldots+n_{q}$. С другой стороны, имеется естественный сюръективный морфизм комплексов $\mathcal{F}_{l_{p}, \ldots, l_{1} ; n_{0} ; n_{1}, \ldots, n_{q}} \rightarrow \mathcal{E}_{l_{p}, \ldots, l_{1} ; n_{0}, \ldots, n_{q}}$. Мы покажем, что гомологии ядра этого отображения сосредоточены в гомологических градуировках $n_{0}+\cdots+n_{q}$ и $n_{0}+\cdots+n_{q}-1$; из этого будет следовать, что гомологии комплекса $\mathcal{E}_{l_{p}, \ldots, l_{1} ; n_{0}, \ldots, n_{q}}$ сосредоточены в градуировке $n_{0}+\cdots+n_{q}$.

В самом деле, пусть $\mathcal{F}_{l_{p}, \ldots, l_{1} ; n_{0} ; n_{1}, \ldots, n_{q}}^{\prime}$ - подкомплекс в $\mathcal{F}_{l_{p}, \ldots, l_{1} ; n_{0} ; n_{1}, \ldots, n_{q}}$, состоящий из всех компонент, в которых у тензорного сомножителя $\mathcal{Z}_{l_{p}, \ldots, l_{1}, n_{0}^{\prime}, \ldots, n_{q^{\prime}}^{\prime}}$ все индексы положительны (т.е. $n_{0}^{\prime} \geqslant 1$ ). Тогда факторкомплекс комплекса $\mathcal{F}_{l_{p}, \ldots, l_{1} ; n_{0} ; n_{1}, \ldots, n_{q}}$ по $\mathcal{F}_{l_{p}, \ldots, l_{1} ; n_{0} ; n_{1}, \ldots, n_{q}}^{\prime}$ изоморфен прямой сумме тензорных произведений компоненты внутренней градуировки $n$ приведенного бар-комплекса алгебры $A$ (с тривиальными коэффициентами) и комплекса $\mathcal{E}_{l_{p}, \ldots, l_{1} ; n_{0}-n, n_{1}, \ldots, n_{q}}$. Из предположения индукции следует, что ядро отображения

$$
\mathcal{F}_{l_{p}, \ldots, l_{1} ; n_{0} ; n_{1}, \ldots, n_{q}} / \mathcal{F}_{l_{p}, \ldots, l_{1} ; n_{0} ; n_{1}, \ldots, n_{q}}^{\prime} \longrightarrow \mathcal{E}_{l_{p}, \ldots, l_{1} ; n_{0}, \ldots, n_{q}}
$$

имеет гомологии только в гомологической градуировке $n_{0}+\cdots+n_{q}$.

С другой стороны, комплекс $\mathcal{F}_{l_{p}, \ldots, l_{1} ; n_{0} ; n_{1}, \ldots, n_{q}}^{\prime}$ имеет конечную возрастающую фильтрацию, индексами которой служат суммы $n_{1}^{\prime}+\cdots+n_{q^{\prime}}^{\prime}$ индексов тензорных сомножителей $\mathcal{Z}_{l_{p}, \ldots, l_{1}, n_{0}^{\prime}, \ldots, n_{q^{\prime}}^{\prime}}$ Присоединенные факторы этой фильтрации суть 
компоненты внутренней градуировки $n$ бар-комплексов алгебры $A$ с коэффициентами в комплексах градуированных $A$-модулей $\bigoplus_{l_{0}=1}^{\infty} \mathcal{E}_{l_{p}, \ldots, l_{1}, l_{0} ; n_{0}-n, n_{1}, \ldots, n_{q}}$. Из предположения индукции следует, что они квазиизоморфны компонентам внутренней градуировки $n$ бар-комплексов алгебры $A$ с коэффициентами в градуированных модулях

$$
\bigoplus_{l_{0}=1}^{\infty} H_{n_{0}+\cdots+n_{q}-n}\left(\mathcal{E}_{l_{p}, \ldots, l_{1}, l_{0} ; n_{0}-n, n_{1}, \ldots, n_{q}}\right),
$$

помещенных в гомологическую градуировку $n_{0}+\cdots+n_{q}-n$. Согласно предположению о модульной кошулевости в формулировке теоремы, их гомологии сосредоточены в гомологической градуировке $n_{0}+\cdots+n_{q}-1$. (Cp. [4, доказательство теоремы 6.1].)

Наконец, вернемся к случаю, когда $\mathcal{Z}_{n_{1}, \ldots, n_{m}}=Z_{n_{1}} \widehat{\otimes} \cdots \widehat{\otimes} Z_{n_{m}}$ есть квазиалгебра замкнутых форм на формальном, комплексно-аналитическом или вещественно-гладком диске, определенная в 2 . Квазиумножение на $\mathcal{Z}$ определяется с помощью диагонального вложения $\Delta_{D}: D \rightarrow D \times D$. Векторное пространство $H_{n_{1}+\cdots+n_{q}}\left(\mathcal{E}_{l_{p}, \ldots, l_{0} ; n_{1} \ldots, n_{q}}\right)$ описывается как пространство замкнутых форм на $D^{p+1+n_{1}+\cdots+n_{q}}$ степеней $l_{1}, \ldots, l_{0}$ по первым $p+1$ группам переменных и 1 по каждой из последних $n_{1}+\cdots+n_{q}$ групп, регулярных вне гиперплоскостей $D^{t} \times L_{s} \times D^{p+1+n_{1}+\cdots+n_{q}-t}$ и имеющих не более чем логарифмические особенности вдоль этих гиперплоскостей, с зануляющимися обратными образами относительно отображений

$$
\operatorname{Id}_{D}^{p+n_{1}+\cdots+n_{t-1}+s} \times \Delta_{D} \times \operatorname{Id}_{D}^{n_{t}-s-1+n_{t+1}+\cdots+n_{q}}: D^{p+n_{1}+\cdots+n_{q}} \longrightarrow D^{p+1+n_{1}+\cdots+n_{q}}
$$

для всех $1 \leqslant s \leqslant n_{t}-1$.

Чтобы показать, что такие пространства замкнутых форм удовлетворяют условию модульной кошулевости из теоремы 2, достаточно рассмотреть их как пространства замкнутых форм степени $l_{0}$ на диске $D$, координатами в котором служит $(p+1)$-я группа переменных, зависящих от параметров, в роли которых выступают переменные из всех остальных групп. Следует включить такие параметры в утверждение теоремы 1 и использовать лемму Пуанкаре с параметрами (доказываемую с помощью обычной процедуры интегрирования, которая сохраняет условия, наложенные на зависимость наших форм от параметров).

Поэтому квазиалгебра $\mathcal{Z}$ кошулева, и квадратично двойственная к ней квазикоалгебра $\mathcal{C}$, описанная в $\S 2$, также кошулева. Кроме того, $\mathcal{Z}$ и $\mathcal{C}$ очевидным образом являются квазиалгеброй и квазикоалгеброй с внешним умножением, и их структуры внешнего умножения соответствуют одна другой при кошулевой двойственности.

\section{ЛитерАтУРА}

[1] A. Beilinson, V. Ginzburg, W. Soergel, Koszul duality patterns in representation theory, J. Amer. Math. Soc., 9:2 (1996), 473-527.

[2] A. Levin, Note on $\mathbb{R}$-Hodge-Tate sheaves, Max Planck Institute for Mathematics Preprint, MPIM2001-37; http://www.mpim-bonn.mpg.de/preprints.

[3] L. Positselski, A. Vishik, Koszul duality and Galois cohomology, Math. Res. Lett., 2:6 (1995), 771-781; http://arxiv.org/abs/alg-geom/9507010. 
[4] L. Positselski, Koszul property and Bogomolov's conjecture, Int. Math. Res. Notices, 2005:31 (2005), 1901-1936.

[5] A. Polishchuk, L. Positselski, Quadratic Algebras, University Lecture Series, vol. 37, Amer. Math. Soc., Providence, RI, 2005.

[6] S. Priddy, Koszul resolutions, Trans. Amer. Math. Soc., 152:1 (1970), 39-60.

Факультет математики и лаборатория алгебраической геометрии НИУ ВШЭ Институт проблем передачи информации РАН e-mail: posic@mccme.ru
Поступило в редакцию 13 августа 2010 г. 